\title{
Seeing number using texture: How summary statistics account for reductions in perceived numerosity in the visual periphery
}

\author{
Benjamin Balas ${ }^{1}$
}

Published online: 9 September 2016

(C) The Psychonomic Society, Inc. 2016

\begin{abstract}
Peripheral visual perception is characterized by reduced information about appearance due to constraints on how image structure is represented. Visual crowding is a consequence of excessive integration in the visual periphery. Basic phenomenology of visual crowding and other tasks have been successfully accounted for by a summary-statistic model of pooling, suggesting that texture-like processing is useful for how information is reduced in peripheral vision. I attempt to extend the scope of this model by examining a property of peripheral vision: reduced perceived numerosity in the periphery. I demonstrate that a summary-statistic model of peripheral appearance accounts for reduced numerosity in peripherally viewed arrays of randomly placed dots, but does not account for observed effects of dot clustering within such arrays. The model thus offers a limited account of how numerosity is perceived in the visual periphery. I also demonstrate that the model predicts that numerosity estimation is sensitive to element shape, which represents a novel prediction regarding the phenomenology of peripheral numerosity perception. Finally, I discuss ways to extend the model to a broader range of behavior and the potential for using the model to make further predictions about how number is perceived in untested scenarios in peripheral vision.
\end{abstract}

Keywords Summary statistics $\cdot$ Numerosity $\cdot$ Peripheral vision

Benjamin Balas

Benjamin.balas@ndsu.edu

1 Department of Psychology, Center for Visual and Cognitive Neuroscience, North Dakota State University, 1210 Albrecht Blvd., Fargo, ND 58108-6050, USA
Peripheral vision is subject to lossy encoding such that substantial information content is not available to observers. Basic examples of how information is lost due to the constraints imposed by visual physiology are the reductions in acuity and color sensitivity in peripheral vision. Peripheral visual acuity is poor largely due to changes in photoreceptor size as eccentricity increases (Virsu \& Rovamo, 1979) and, similarly, peripheral color sensitivity is reduced as a direct result of the decreasing proportion of cones versus rods as retinal eccentricity increases (Martin, Lee, White, Solomon, \& Rüttiger, 2001).

Beyond these initial limitations, downstream constraints on peripheral vision further limit the fidelity of the visual system's representation of visual structure. In particular, the phenomenology of visual crowding (Whitney \& Levi, 2011) is an important example of how perception is limited by processes that further reduce representational fidelity in peripheral vision. Visual crowding refers to deficits in peripheral target recognition induced by neighboring flanking items. Crowding does not impair target detection, which differentiates crowding from masking (Pelli, Palomares, \& Majaj, 2004), and observers' ability to identify isolated peripheral targets rules out accounts based on visual acuity in the periphery. Crowding thus results from additional constraints on visual processing that limit representational fidelity. Understanding the nature of these constraints and determining how they limit visual processing in computational terms is an ongoing enterprise. A range of factors alter crowding strength, and the effects of manipulating flanker/target similarity (Bernard \& Chung, 2011), the geometric arrangement of flankers relative to targets (Manassi, Sayim, \& Herzog, 2012), and other spatial and temporal factors that influence performance (Kooi, Toet, Tripathy, \& Levi, 1994), all offer important constraints on the computational mechanisms that lead to crowding, and by extension, may also govern a wide 
range of phenomena observed in peripheral vision (Rosenholtz, 2011). Here, I consider how these mechanisms may govern the perception of numerosity in peripheral vision using a recent model of peripheral visual function to explain recent empirical data.

A key issue in understanding how peripheral vision works is characterizing the nature of integration in peripheral vision. A fundamental assumption of most descriptions of crowding and related phenomena is that image structure is somehow pooled over increasingly large chunks of the visual field as eccentricity increases. In the context of crowding, the size of the regions in which crowding occurs scales with eccentricity (Pelli \& Tillman, 2008; Rosen, Chakravarthi \& Pelli, 2014), a relationship referred to as Bouma's law (Bouma, 1973). This is a quantitative description of where information is pooled, but it does not tell us how information is pooled. Subjectively, peripherally viewed image features often appear jumbled or mixed together, but what is the nature of that jumbling and mixing? What processes lead to a description of image structure such that visual features appear to be rearranged and combined in strange ways? One proposal is that image structure may be represented by summary statistics that describe visual features using distributional information (e.g., texture descriptors), leading to a histogram-like representation of appearance that is sufficient to capture some aspects of what objects or targets are present in a peripheral region, but that lacks joint encoding of form and location. In general, models based on summary statistics record some information about what features were present in a stimulus, but tend to lack information about where those features were and how they co-occurred spatially with other features. Initially, summarystatistic models of crowding used simple distributional measurements to account for behavior. For example, Parkes, Lund, Angelucci, Solomon, and Morgan, (2001) suggested that stimulus averaging within a crowded array could explain observers' responses in an orientation discrimination task. Rather than representing each item in a crowded array, they proposed that observers were subject to compulsory averaging of the orientations in the stimulus and made responses based on that average. Although adequate for simple scenarios (e.g., oriented bars), it is difficult to understand exactly what averaging would mean for an array of complex objects (Wallace \& Tjan, 2011). Recently, texture-based summary-statistic models that address this issue have been applied to a range of problem domains. Such models rely on texture features to describe image structure and are applicable to arbitrary image inputs. Also, using models that support texture synthesis makes it possible to test model predictions behaviorally using "mongrel" images that reflect the image constraints imposed by the statistical descriptors imposed by the model. If the model captures the information loss imposed by peripheral vision, then performance with original images presented in the periphery should match performance with mongrels. In several recent reports, performance with mongrels generated using the Portilla-Simoncelli texture synthesis algorithm (Portilla \& Simoncelli, 2000) correlates significantly with performance in peripheral tasks, suggesting this is a useful model of peripheral encoding. For example, Balas, Nakano, and Rosenholtz (2009) demonstrated that mongrels could account for performance across a range of visual crowding tasks. Visual search performance, which depends on observers' ability to identify candidate target locations in the periphery, is also accounted for by mongrels that reflect local summary statistics (Rosenholtz, Huang, Raj, Balas, \& Ilie, 2012). More broadly, the texture statistics in the Portilla-Simoncelli model may be a good approximation of midlevel computations carried out in area V2 (Freeman, Ziemba, Heeger, Simoncelli, \& Movshon, 2013) used to encode scene structure across the visual field (Freeman \& Simoncelli, 2011). This set of summary statistics is thus a promising candidate model of peripheral visual function that may explain performance in many tasks.

In this study I have applied the model to numerosity perception in the visual periphery, which is an interesting problem domain for several reasons. Perceived numerosity in large arrays of items appears to depend upon texture-like properties of the array including density and local area computations (Durgin, 2008; Raphael, Dillenburger, \& Morgan, 2013), suggesting that texture features may be appropriate descriptors of array appearance. Though numerosity judgments are not necessarily texture based in all settings (Mandler \& Schebo, 1982; Palomares, Smith, Pitts, \& Carter, 2011), texture representations do appear to be employed for numerosity judgments as a function of stimulus features such as array density. For example, Anobile, Cicchini, and Burr (2014) demonstrated that when element density is relatively low, numerosity discrimination thresholds have a constant Weber fraction (linear increases as a function of numerosity). However, when density is comparatively high, numerosity discrimination thresholds increase according to a square-root law, which suggests a different, texture-like mechanism for low- versus highdensity arrays.

Perceived numerosity has also been shown to change as a function of peripheral viewing in ways that are consistent with visual crowding. The aforementioned transition between linear versus square-root threshold increases as a function of numerosity occurs at different dot densities as a function of eccentricity-for example, such that texture mechanisms appear to be used at lower densities in the periphery (Anobile, Turi, Cicchini, \& Burr, 2015). This dependence on eccentricity is consistent with the impact of visual crowding on other tasks, and the square-root dependence between discrimination thresholds and numerosity further supports the use of texture descriptors as a tool for understanding peripheral numerosity in particular. Moreover, other unique features of how number is perceived in the periphery are 
intriguing targets for a model of peripheral vision. Specifically, Valsecchi, Toscani, and Gegenfurtner (2013) showed that the numerosity of peripherally viewed arrays of dot is reduced relative to numerosity perceived in the fovea. They reported that numerosity was reduced by approximately $60 \%$ to $80 \%$, depending on the specific stimulus configuration and eccentricity used in their experiments, suggesting that the appearance code that supports peripheral visual processing represents the array in a way that number is consistently reduced. By manipulating various stimulus properties including the minimum distance between elements, the authors demonstrated that visual crowding may drive this effect. Given the success of explaining visual crowding via specific summary statistics and the evidence indicating that crowding contributes to behavioral performance in peripheral numerosity perception, I therefore chose to investigate whether the Portilla-Simoncelli texture statistics could account for the reduction in perceived numerosity reported by Valsecchi et al. (2013).

Specifically, I focused on examining whether parameter values that were sufficient to explain observers' performance in previously reported visual crowding tasks (Balas et al., 2009) yielded numerosity underestimates when the model was presented with arrays similar to those used in Valsecchi et al. (2013). In particular, I chose to examine whether the model could account for the amount of underestimation when randomly placed dots were presented to observers in the periphery and also whether the model would exhibit similar sensitivity to varying interdot distance. Overall, these simulations reveal that decreased numerosity emerges naturally from the model in all conditions. However, while the quantitative fit is good when randomly placed dots are used as target stimuli, the model does not exhibit sensitivity to interdot distance that is consistent with human performance. Despite this shortcoming, I also demonstrate that the model exhibits sensitivity to element shape, which represents a novel prediction regarding peripheral numerosity perception that remains to be tested in human participants. These particular summary statistics thus offer limited explanatory power for understanding peripheral numerosity perception, which constrains the scope of this model of peripheral vision. Nonetheless, I argue that the model may still suggest interesting directions for future research and also discuss principled extensions of the model that may reveal critical properties of peripheral vision that must be adequately characterized to increase the generality of summary-statistic models of the periphery.

\section{Method}

In the simulations I report here, my aim was to examine three distinct aspects of peripheral numerosity perception, two of which were reported by Valsecchi et al. (2013) and a third scenario that has not been tested psychophysically. With regard to the former, I carried out simulations to determine (1) if peripheral numerosity would be underestimated in "mongrel" images created using the Portilla-Simoncelli algorithm, and (2) if varying the minimum interdot distance between elements in a peripherally viewed array would affect underestimation in mongrel images. With regard to the latter, I carried out additional simulations to determine if changing element shape (e.g., dots vs. crosses/circles) led to changes in numerosity underestimation. In general, the model supports quantitative measurement of the amount of underestimation, allowing direct comparison between human observers and the model.

\section{Creating dot arrays}

To generate the arrays that mongrel images would be based on, each simulation began by plotting randomly placed elements using basic graphing routines in MATLAB. To examine peripheral numerosity subject to random element placement (Experiment 1), element positions were sampled from a regular grid spanning zero to 255 units in both the $x$ - and $y$-axes, with element centers spaced 10 units apart (to eliminate overlap) within a bounding frame 10 units wide (to eliminate elements being cut off during plotting). Each dot was then randomly perturbed horizontally and vertically by sampling $x$ and $y$ displacements independently from a normal distribution with zero mean and a standard deviation of 2 units. To examine the impact of clustering (varying interelement distance) on peripheral numerosity (Experiment 2), element positions were sampled according to a satellite algorithm (Allik \& Tuulmets, 1991) that guarantees that each newly sampled element is positioned within some fixed distance from a previously plotted element. Finally, to examine the potential effects of element shape on numerosity perception (Experiment 3), the markers used to plot randomly selected points could either be dots (closed circles), crosses, or naughts (open circles). In all cases, the MATLAB plot depicting all of the dots was resized (if necessary) to $256 \times 256$ pixels before texture analysis and synthesis.

\section{Synthesizing "mongrels" from dot arrays}

To create a mongrel from each simulated dot array, I applied the Portilla-Simoncelli texture synthesis algorithm to each image. This involves first using analysis routines to describe the target image using a set of joint wavelet statistics. The analysis algorithm includes free parameters that specify the number of spatial scales ( $\mathrm{N}$ sc) used to compute wavelet coefficients, the number of orientation bands ( $\mathrm{N}$ or) used to describe image structure, and the size of the spatial neighborhood (N_neigh) over which local correlations are computed. I 
chose these parameter values to match those reported in Balas et al. (2009) to test the hypothesis that values that proved adequate for accounting for visual crowding may also account for numerosity underestimation in the periphery (N_sc $=4$, N_or $=4$, N_neigh $=9$ ). Following texture analysis, synthesis proceeded by generating a random starting image and adjusting this image iteratively until it matched the target image's statistics (see Fig. 1).

\section{Counting dots in original and synthetic arrays}

To objectively measure numerosity in each dot array, I applied morphological operators to thresholded versions of the original and synthetic arrays generated for each simulated trial. The synthetic arrays were histogram-matched to the parent image used to generate them, and an intensity threshold was applied to both images to convert them to binary images. Both images consistently had bimodal intensity histograms with narrow peaks centered near intensity values of 2 (near-black) and 200 (near-white), making it possible to impose a threshold in between these values without concern that the outcome depended critically on the exact intensity threshold used. Next, I applied MATLAB functions for labeling contiguous regions to obtain the size of each black region in both the original and synthetic image. Each original and synthetic array was thus described in terms of the number of segmented regions in the thresholded image. Regions in the synthetic array were counted only if they were at least as large as the smallest region in the original array. In keeping with the original report by Valsecchi et al. (2013), I expressed model performance using the ratio of dot numerosity in the synthetic image relative to its parent. Perfect performance would yield a ratio of 1 , while overestimates would be larger and underestimates smaller than this. For each combination of stimulus parameters (target numerosity, clustering conditions) I conducted 50 simulations to obtain mean underestimation ratios and bootstrap estimates of $95 \%$ confidence intervals.
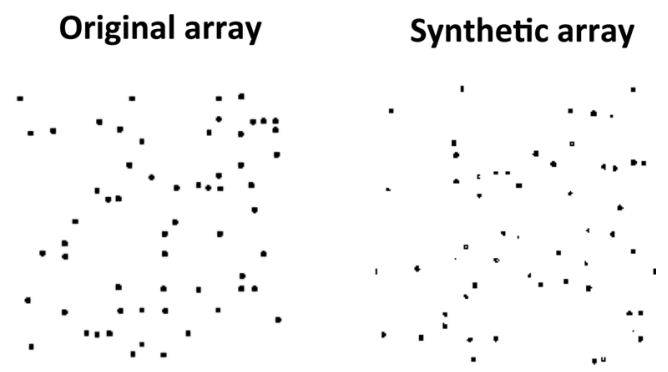

Fig. 1 An example of an original dot array and a synthetic array created from this parent image. These images have been processed per the description in the text, which includes resizing of the original array and thresholding of both the original and synthetic arrays to yield a binary image

\section{Results}

To summarize the results of the model, I briefly describe the parameters that were allowed to vary across the three experimental scenarios considered here, and report underestimation ratios for all conditions as compared to the results reported in Valsecchi et al. (2013).

\section{Experiment 1-Random dot placement}

In these simulations, target numerosity was either 30,60 , or 90 dots, and elements were randomly positioned in the original arrays. Dot size was set to a MATLAB marker size of 18 units. The observed underestimation ratios for each target numerosity (with $95 \%$ confidence intervals) are listed in Table 1.

These ratios exhibit the stability across target numerosities reported in Valsecchi et al. (2013) and are quantitatively a good fit to their results from the 12-degree eccentricity condition in their Experiment 1 (these means vary between $79 \%$ and $85 \%$ across the same target numerosities).

\section{Experiment 2-Clustering}

In these simulations, target numerosity was either 20,40 , or 60 dots. For each target numerosity, the minimum allowed interdot distance was controlled via "satellite" sampling to yield "small," "medium," and "large" clustering. In Table 2, I have listed the mean underestimation ratio for each condition along with $95 \%$ confidence intervals. At best, the model exhibits only a mild sensitivity to clustering (an increase of approximately $5 \%$ between the small and large clustering conditions), which is far smaller than the approximately $30 \%$ difference reported in Valsecchi et al.'s (2013) Experiment 2. Clustering thus matters somewhat to the model, but not nearly as much as it matters to human observers.

\section{Experiment 3-Element shape}

In these final simulations using Portilla-Simoncelli mongrels (which have not been tested psychophysically), target numerosity was either 40 or 60 elements. For each numerosity, target shape was either dots (closed circles) 18 units in size,

Table 1 Mean underestimation ratios for dots as a function of target numerosity

\begin{tabular}{ll}
\hline & Mean underestimation ratio \\
\hline 30 dots & $85.2 \%[82 \%, 88 \%]$ \\
60 dots & $85.4 \%[83 \%, 87 \%]$ \\
90 dots & $86.0 \%[84 \%, 87 \%]$ \\
\hline
\end{tabular}

Values in brackets represent $95 \%$ confidence intervals of the mean 
Table 2 Mean underestimation ratios as a function of interelement distance (clustering) and target numerosity

\begin{tabular}{lllr}
\hline & 20 elements & 40 elements & 60 elements \\
\hline Small & $80.2 \%[75 \%, 86 \%]$ & $81.0 \%[79 \%, 83 \%]$ & $84.4 \%[82 \%, 87 \%]$ \\
Medium & $84.6 \%[72 \%, 99 \%]$ & $83.3 \%[77 \%, 87 \%]$ & $83.0 \%[80 \%, 85 \%]$ \\
Large & $86.4 \%[84 \%, 89 \%]$ & $86.5 \%[82 \%, 88 \%]$ & $86.5 \%[85 \%, 89 \%]$ \\
\hline
\end{tabular}

Values in brackets represent $95 \%$ confidence intervals of the mean

crosses that were eight units in size, or naughts (open circles) that were also eight units in size. The marker size of naughts and crosses was selected so that element size in the original arrays would be matched when naughts and crosses were drawn with a line of sufficient thickness to guarantee that elements in the original images were counted correctly using our morphological operators. The mean underestimation ratio for each condition with associated $95 \%$ confidence intervals is listed in Table 3. At both target numerosities, underestimation varies with shape: Crosses lead to greater underestimation than dots, and naughts lead to even greater underestimation.

\section{Image blur as an alternate model of peripheral numerosity}

Finally, to assess whether or not increased image blur in peripheral vision could also account for the results, I carried out additional simulations in which I generated "blur mongrels" made by convolving the original dot arrays with Gaussian filters with increasingly large spatial kernels. In these simulations, I observed a clear effect of target numerosity on the underestimation ratio - for numerosities of 30 or 60 dots, even severe blur tended to yield underestimation ratios of approximately $90 \%$, while the same amount of blur applied to an array of 90 dots yielded a much larger underestimation ratio of approximately $15 \%$ to $20 \%$. These effects were further exacerbated when clustered dots were used because small interdot distance could lead to arrays that were dramatically underestimated due to the vast majority of dots being blurred together. This discrepancy between an extreme effect of target numerosity on "blur mongrels" and the lack of such an effect in human observers suggests that reduced numerosity is likely not the result of blur, which is consistent with arguments made in prior reports.

Table 3 Mean underestimation ratios as a function of element shape and target numerosity

\begin{tabular}{lll}
\hline & 40 elements & 60 elements \\
\hline Dots & $85.3 \%[84 \%, 86 \%]$ & $85.9 \%[84 \%, 87 \%]$ \\
Crosses & $76.8 \%[73 \%, 78 \%]$ & $78.0 \%[75 \%, 80 \%]$ \\
Naughts & $66.8 \%[64 \%, 70 \%]$ & $66.6 \%[65 \%, 68 \%]$ \\
\hline
\end{tabular}

Values in brackets represent $95 \%$ confidence intervals of the mean

\section{Discussion}

These simulations demonstrate that some (but not all) aspects of peripheral numerosity perception emerge naturally from a summary-statistic model of peripheral vision. This is consistent with an emerging computational description of peripheral vision in terms of the constraints imposed by a texture-like statistical code for appearance (Rosenholtz, 2011) and also with the proposal that perceived numerosity depends on crowding and texture statistics in some settings. The model is limited in scope, however. Although performance when dots are placed randomly is quantitatively good, it fails to reproduce the phenomenology associated with dot clustering. This latter outcome complicates matters a good deal, because the psychophysical impact of clustering (and related results concerning element density) is an important piece of supporting evidence for the putative relationships being explored here between peripheral vision, visual crowding, texture representations, and numerosity perception. I conclude by discussing the implications of the limited success of the model first, then consider how the model may be extended in a principled fashion to account for a broader range of data.

To speak first of what the model can do, like prior reports describing correspondences between summary-statistic codes for appearance and the perception of peripheral targets, I find that the basic phenomenology of a task that depends on peripheral vision is a natural consequence of using texture features to describe discrete objects: Mongrels underestimate numerosity. Critically, the observation that the same parameter values that predict visual crowding performance across a range of conditions also accounts for perceived numerosity in arrays of randomly placed dots (Experiment 1) suggests that at least in some cases, numerosity judgments are affected by the information lost due to the imposition of an image-based texture representation of image structure. An advantage of this model is that it does not require an explicit description of items in an array in terms of their position or "occupancy" (Allik \& Tuulmets, 1991), nor does it depend on special computations that capture numerosity/density (Dakin et al., 2011). Instead, the model depends on a general texture-based description of appearance that can be applied to arbitrary inputs. This does not preclude specific mechanisms for number processing that operate at later stages of visual cognition, but does offer a means of accounting for some properties of number perception 
in terms of lower-level processes. This model also succeeds where some simpler ones fail: Estimating numerosity after blurring dot arrays has a tendency to lead either to catastrophic amounts of underestimation as clustering or target numerosity increases or to very little underestimation at all.

But what to make of the failure to reproduce the effects of clustering? This obviously signals that this model (which has been successful in many domains) fails to capture some feature of peripheral vision or numerical cognition. That the model has such a limit is not surprising, and delineating the boundaries of the model's ability to account for behavior is an important step towards developing better theories of peripheral vision. Though mongrel-like images of natural scenes have been described as metameric stimuli that make it possible to assay specific stages of processing along the ventral visual stream (Freeman \& Simoncelli, 2011), it is also the case that adult observers are quite sensitive to discrepancies between natural images and synthetic images made from them (Balas, 2006; Wallis, Bethge, \& Wichmann, 2016). Portilla-Simoncelli mongrels are thus known to not capture all the information observers have access to in peripheral vision, and here we are likely seeing a consequence of that imperfect representation. Applying full-field texture synthesis with multiple, overlapping pooling regions (per Freeman \& Simoncelli, 2011) may be one way to extend the scope of the current model in a principled fashion, and perhaps this may account for a wider range of phenomena in this domain. Alternatively, it may also be necessary to include a wider class of texture descriptors (e.g., center-surround descriptors) to arrive at a more expressive vocabulary for peripheral image structure. Of course, it may also be the case that there are simply other mechanisms at work here, and this is as far as summary statistics will take us in terms of understanding how numerosity is perceived.

With regard to this latter possibility, however, I want to emphasize the novel results included here regarding the impact of shape on underestimation. To my knowledge, this outcome has not been observed experimentally and contradicts the overall characterization of number perception as being sufficiently abstract to be robust to manipulations of element shape or orientation. Although the current model clearly has limits, its moderate success also argues for its utility as a means of generating novel hypotheses regarding peripheral vision. My results suggest in part that we need a better model of the periphery to account for some of the ways people perceive number, but they also suggest that we can potentially learn more about how peripheral vision in general (and number perception in particular) works by querying appearancebased models such as this one. I conclude, therefore, that although summary-statistic representations of the periphery cannot currently get us all the way toward a comprehensive account of peripheral numerosity, they offer enough explanatory power to be an important platform for further exploration of peripheral visual function.
Acknowledgements This research was supported by NIH Grant EY024375-01 awarded to B. B. and NIGMS Grant P20 103505 supporting the Center for Visual and Cognitive Neuroscience at NDSU. Thanks also to Ruth Rosenholtz and Thomas Wallis for helpful comments about the counting procedures used to estimate numerosity in synthetic arrays.

\section{References}

Allik, J., \& Tuulmets, T. (1991). Occupancy model of perceived numerosity. Perception \& Psychophysics, 49, 303-314.

Anobile, G., Cicchini, G. M., \& Burr, D. C. (2014). Separate mechanisms for perception of numerosity and density. Psychological Science, 25, 265-270.

Anobile, G., Turi, M., Cicchini, G. M., \& Burr, D. C. (2015). Mechanisms for perception of numerosity or texture-density are governed by crowding-like effects. Journal of Vision, 15(5), 1-12.

Balas, B. (2006). Texture synthesis and perception: Using computational models to study texture representations in the human visual system. Vision Research, 46, 299-309.

Balas, B., Nakano, L., \& Rosenholtz, R. (2009). A summary-statistic representation in peripheral vision explains visual crowding. Journal of Vision, 9(12), 13, 1-18.

Bernard, J. B., \& Chung, S. T. (2011). The dependence of crowding on flanker complexity and target-flanker similarity. Journal of Vision, 5(11), 1-16.

Bouma, H. (1973). Visual interference in the parafoveal recognition of initial and final letters of words. Vision Research, 13, 762-782.

Dakin, S. C., Tibber, M. S., Greenwood, J. A., Kingdom, F. A. A., \& Morgan, M. J. (2011). A common visual metric for approximate number density. Proceedings of the National Academy of Sciences, USA, 108, 19552-19557.

Durgin, F. H. (2008). Texture density adaptation and visual number revisited. Current Biology, 18, 855-856.

Freeman, J., \& Simoncelli, E. P. (2011). Metamers of the ventral stream. Nature Neuroscience, 14, 1195-1201.

Freeman, J., Ziemba, C. M., Heeger, D. J., Simoncelli, E. P., \& Movshon, J. A. (2013). A functional and perceptual signature of the second visual area in primates. Nature Neuroscience, 16(7), 974-981.

Kooi, F. L., Toet, A., Tripathy, S. P., \& Levi, D. M. (1994). The effect of similarity and duration on spatial interaction in peripheral vision. Spatial Vision, 8, 255-279.

Manassi, M., Sayim, B., \& Herzog, M. H. (2012). Grouping, pooling, and when bigger is better in visual crowding. Journal of Vision, 12(10), $1-14$.

Mandler, G., \& Schebo, B. J. (1982). Subitizing: An analysis of its component processes. Journal of Experimental Psychology: General, $111,1-22$.

Martin, P. R., Lee, B. B., White, A. J., Solomon, S. G., \& Rüttiger, L. (2001). Chromatic sensitivity of ganglion cells in the peripheral primate retina. Nature, 410, 933-936.

Palomares, M., Smith, P. R., Pitts, C. H., \& Carter, B. (2011). The effect of viewing eccentricity on enumeration. PLOS ONE, 6, e20779.

Parkes, L., Lund, J., Angelucci, A., Solomon, J. A., \& Morgan, M. (2001). Compulsory averaging of crowded orientation signals in human vision. Nature Neuroscience, 4, 739-744.

Pelli, D. G., Palomares, M., \& Majaj, N. J. (2004). Crowding is unlike ordinary masking: Distinguishing feature integration from detection. Journal of Vision, 4(12), 1136-1169.

Pelli, D. G., \& Tillman, K. A. (2008). The uncrowded window of object recognition. Nature Neuroscience, 11, 1129-1135. 
Portilla, J., \& Simoncelli, E. (2000). A parametric texture model based on joint statistics of complex wavelet coefficients. International Journal of Computer Vision, 40, 49-71.

Raphael, S., Dillenburger, B., \& Morgan, M. (2013). Computation of relative numerosity of circular dot textures. Journal of Vision, 13(2), 1-11.

Rosen, S., Chakravarthi, R., \& Pelli, D. G. (2014). The Bouma law of crowding revised: Critical spacing is equal across parts, not objects. Journal of Vision, 14(6), 1-15.

Rosenholtz, R. (2011). What your visual system sees where you are not looking. Proceedings of SPIE: Human Vision and Electronic Imaging, XVI, 1-6.

Rosenholtz, R., Huang, J., Raj, A., Balas, B., \& Ilie, L. (2012). A summary statistic representation in peripheral vision explains visual search. Journal of Vision, 12(4), 1-17.
Valsecchi, M., Toscani, M., \& Gegenfurtner, K. R. (2013). Perceived numerosity is reduced in peripheral vision. Journal of Vision, 13(13), 1-16.

Virsu, V., \& Rovamo, J. (1979). Visual resolution, contrast sensitivity, and the cortical magnification factor. Experimental Brain Research, 37, 475-494.

Wallace, J. M., \& Tjan, B. S. (2011). Object crowding. Journal of Vision, 11(6), 1-17.

Wallis, T. S. A., Bethge, M., \& Wichmann, F. A. (2016). Testing models of peripheral encoding using metamerism in an oddity paradigm. Journal of Vision, 16(2), 1-30.

Whitney, D., \& Levi, D. M. (2011). Visual crowding: A fundamental limit on conscious perception and object recognition. Trends in Cognitive Science, 15(4), 160-168. 